\title{
Comunicación y Semiosis para la prevención de minas antipersonal, Colombia un caso
}

\author{
MIREYA BARÓN PULIDO \\ Politecenico Grancolombiano \\ mbaronpunpoliedu.co
}

\begin{abstract}
Magister en Lingulistica Española, Instituto Caro y Cuerva. Langue Française, Paris IV-Sorbonne. Docente Inwestigadora Tiempo Completo de la Faxultad de Mercadeo, Comunicación y Artes, del Politéconico Grancolombiano, adscrita al Departamento de Cumunicación. Lider del Gruplac crec Comunicación Estratégica y Creativa. Conferencista de agendas internacionales: ccrescom. Bolivia 2007, Ascciación Latinoamericana de Investigación en Comunicación alaic-Méxoico 2008, Asociación Atgentina de Semiótica AAS 2010. Publicaciones en revistas intemacionales Razón y Palabra, Foro Iberoamericano sobre Estrategias de Comunicación (Fisec). Membreclas: Red de investigación en comunicación en Colombia (Redicom), Centro de Investigación Colombia Regional y Empresarial del Politécnico Grancolombiano (Cicre).
\end{abstract}

\section{Resumen}

El arificulo muestra los resultados de investigación' de los elementas que desde la narrativa audisovisual caracterizan el grado de presencia/ausencia de actores y nt veles de mocmarion que relatan el flagelo de las Minsis Antipersonal Es una investigacion de indole cratitativa que compila el Análisis de Contenido - AC-de 28 plezas aud kovlsuales locales e internecionales y que suricten ta construccion de sentido descte yarios niveles de la informacion ante la población vialnerable dal pass Se compila lis tendencia de compsinamiento tanto de códigos konicos linguísticos, como de los componentes maifologico. semántico y sintartico: analisis que permite evirsenciar 2 tipos de namaciones; La narrada desde pais victima para pobleción themacional y la marrada desde pais no victima para poblacion internacional. De dicha pesquisa surge la creacion del Documental is no la boro no to recola: propuesta comuricativa harrada desde y para la población campesina e infantil del pais, La investigactión confru con respaldo del Departamento $t+D+I$ y con et concurso del semillero de estudtantes Isabella Montalivo (Prograna de Comunicacion Soctal. Reriodisinul. Nathalis Vir Bas y fuan D. Villabona (Medica Audiowisuales)?

\section{Palabras Claves}

Productos audiovisuales, minas antipersonal analisis cte conteridio.

\section{Abstract}

This urticle presents the resenrch resalts from flis audion! stal narrative of the elements that characterizo the degroe

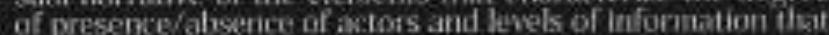
describe the scourge of Antipersonnd Mines This is a gualttative research that commiles line Content Anaksis - Ch-of 28 local and international atdiovisual samples which sug. gest the construction of sensa from various kevels of infoi ination regarding the vulnerable popullation of the countiv The artic le compites the behavior tendency not only of icanic and linguistic coslos but also of morphologic semantic, and syntrictic comenonents This analys is allows eviderocing two types of narrative the one narrated from the victini country to the international population and the one narrated from novictim country to internitional popalation. From this er: senrch, the creatfor of the documentasy "Sr no ka boto. wo 60 reobla, communicative proposa narrated from and for the pcasarn and child popinlation of the couniry, The researchi Was supported by the $4+0+1$ Department and by the contest of studerit secdibed. lsabelin Rontalvo ISocial Communios. tion-Journalison Programi. Nathalia Vargas and Juan D. Vi. Ilabona (Audowisual Mextia).

\section{Key words}

Audiavisual Products. Landimines Antipersonet Content Aralysis.

\footnotetext{
1 El proyecto de investigación ha ssdo financlado por ls Fundación Politécnico Grancolombiano, metiante el contrato de investigación $2010-14-F 00$ -
}

2 Ademas, se destaca que el diserio gráfico tanto del estuche del documental Si no lo bura no Jo recojir, camo bos afiches imagen del bocumental, producte de la actual investigación, fueron elaborados por los estudiantes Yami Nagao y Dayana Navartete. 
Este articulo socializa los restultados de la investigación en su segunda fase, y pretende evidenciar los actores y los niveles de información que sugiere la narrativa audiovisual por medio de uno de sus componentes; la palabra hablada, entendida en los siguientes términos

En la palabra hablada hallamos una gran multiplicidad de sistemas sipnificantes, que enitran en correlactón. Es una multiplicidad que nos refiere a edaxL, región. estado de ánimo y muchas cosas mís. Con la woz tenemos gran cantidad de informacion (Paoli, 2002, p. 276).

El marco conceptual del actual articulo se construye a partis de tres aristas: comunicación y cultura, semiologia de los signos en la narrativa audiovisual, y comunicación y educación. Es decir, partimos de la relación que sugiere la creación e interpretación de sistemas signicas como construcción de sentido para un grupo sociocultural, y que, por ende, puede generar niveles de educación al grupo interlocutor para el cual está pensado el mensaje.

En primer lugar, queremos destacar que la sociedad elabora y legitima jos sistemas simbólicos de cara a las necesidades culturales que sus grupos participantes utilizan e interpretan; por ello el articulo muestra que esta invención de sistertas simbólicos-significantes -en términos de Ferdinand de Saussure, en tanto imágenes acuisticas y visuales- se convierten en el anclaje para estudiar e interpretar la cultura (Sausure, 1945, p. 87).

El corpus objeto de estudio corresponde a la selección del registro audiovisual cuyo relato narra uno de los flagelos que afecta a la sociedad del siglo XXI: minas antipersonal: toda vez que la comunicación y por ende sus productos comunicativos ${ }^{3}$ actuan al superar el simple papel de la información, eintentar la elaboración de sentidos más depurados como la prevención, la educación y el fortalecimiento de acciones que garanticen los derechos humanos en una sociedad.

3. Para el actual articubo, aclaramos que la coounicactony, por ente,

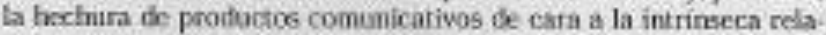
ctón con la sociedad. cosistruyen, emergen y doconstruyen mensaiks acorde con sus recesidades, ibrtalezes y conflictos. En estos termiaos queremos destacar que Ta socledad es geoef sodora de mensajes, de prrpusstas - la comunicación permile que éstas lleguen a todos las sectores, Por tanto, la revolución del año 2000 es bacer de la cominicacion una hetramients accesible para todas, en espocsal para aguellos sectures if la sockedart mis vulnerables" (Átvarez, 2000, p. 4.5).
En segurdo lugar, vale la pena destacar la relación reciproca entre dos aspectoss sistema simbólico y cuinura, esta última elaborada y perceptible a partir del primero, por ello se hace referencia a cultura como

aquel conjunto integrado de formas intelectuales expresidas mediante formas simboilicas, a partir de las cuales se generan y reconocen valores y significados en ba vida social (Paolt, 2002, p. 65).

Precisamente, estas formas simbolicas son reconocidas por cuanto extrapolan aquellas convenciones que permiten a la vez experimentar, comprender y valorar una realidad.

En este contexto, queremos ser enfáticos en que fortalecer contenidos en productos comunicativos para el actual caso y de manera especifica en audiovisuales. e implementar el mensaje de la prevención por medio de testimonios y de rostros de la realidad del pais, se convierten en supuestos neurálgicos de un marco conceptual de la comunicación como estrategia en cuanto acción discursiva desde su papel soclal.

En tercer lugar, reconocemos que la gramática audiovisual se debe concebir, como "toda una praxis de escritura en tanto observadora social" (Pérez, 2007). En estos términos dicha gramática nos ofrece según los participantes, la intención del mensaje y el perfil de la audiencla para la cual está pensado el mensaje. Se deben tener presente los varios tlpos de géneras para productos audiovisuales pasando por el reportaje, la cronica, el ensayo y el documental; inclusive tener en cuenta las posibles hibridaciones en los que se fusionan significantes con alta iconicidad alternando con significantes de naturaleza cualisigno, de acuerdo con Peirce (2007).

A partir de esos referentes, nos sentimos en la necesidad de no olvidar que la elaboración de productos comunicativos en el contexto comunicación y educación, se convierte en uno de los ejes temáticos que preocupan tanto a la agenda local como a la agenda internacional en comunicación. Este es el caso de los diversos grupos de trabajo en América Latina que estudian la Comunicación para el Cambio Social (CSC):

1.) el estudio de las derechos humanos, como marco de desarrollo y "horizonte ético" del cambio social, deben 
ser una prioridad en la agenda soctal y cxsmunicativa larinoamericana slarbo ef prolongalo y ststemático contexto de vulnerarfones, volencla y exclusionon en el que visimos, y la potencla transformadora $y$ autoafirmativa propla que tieste la promoción de derechos. Debikto a que las denchos humanos, el desarrollo y el cambio social san, por definixión, universos réacionales pensamos que la comvunicación (sctuando en sentidio amplio $y$ en conjunto con la antropologia, la ciencin politica, la socjologia y las artesh ocupa in higar prbvleglatio en la construcción de estrateglas. redes, contextus y procesos para el acercamicnto a taies objetives KGumucka-Dagron, Alatc, 2006).

Para comprender la caracterización de la gramática audiovisual en las piezas seleccionadas objeto de estudio, debemos advertir que dada la naturaleza de la porción de realidad a comunicar, esta semiosis narra una comunicación que enfatiza en la información, más que en la prevención, por medio de loonos, simbolas $e$ indices -en términos peirceanos- puestos ante la lente con poco uso de ángulos, pero con diversos matices de planos. Fortalecer contenidos en productos comunicativos audiovisuales, e implementar el mensaje de la prevención por medio de testimonios y rostras de la realidad del pais se convierten en supuestos neurálgicos de un marco conceptual de la comunicación como semiosis de acción discursiva desde su papel social. Esta investigación, se convierte en un espacio epistemológico en el que la comunicación obra como un soporte esencial para asumir dichas realidades con responsabilidad social, educativa y preventiva

Para la apuesta auchovisual gue presentamos como pieza complementarla a nuestra investigación se considera valinso y cportuno que sea el sentir de la voz ciudadana local. el eje que construya el sentido y estructure la narración del documental a elaborar.

A partir de una serie de preguntas objetivo sugeridas por los productores-realizadores del documental. se suele construir el guión de este tipo de producto comunicativa. Tenienda en cuenta esta variante, para este caso se habla de un preguión, por cuanto es a partir de la captura de las entrevistas, de las imágenes y de los escenarios como se compila y dinamiza el guión del documental (Perez, 2007).

\section{Pautas metodológicas}

Es pertinente aclarar que la actual investigación acude a una de los procedimientos metodológicos que desde las ciencias sociales se ha venido utilizando: análisis de contenido (en adelante, se nombrará como AC)). En estos tésminos, se acudio al anál isis del comportamiento discursivo en la comunicación audiowisual a partir del grado de presencia o ausencla de varios tipos de códigos y componentes de la gramática audiovisual:

Tabla 1. Categorias de análisis

\begin{tabular}{|c|c|c|c|}
\hline Tema & Universo & Muestra & $\begin{array}{c}\text { Unidad de } \\
\text { análisis }\end{array}$ \\
\hline 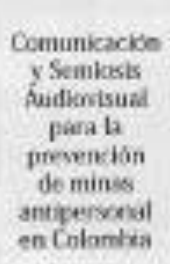 & 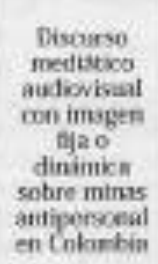 & 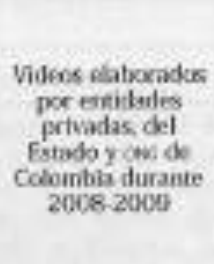 & $\begin{array}{l}\text { Codleo } \\
\text { limguistion } \\
\text { Codigo vksial } \\
\text { Componente } \\
\text { morfokgice } \\
\text { Componente } \\
\text { sints:tico } \\
\text { Componenie } \\
\text { semantion }\end{array}$ \\
\hline
\end{tabular}

Entre las unidades de análisis, se observaron los siguientes rasgos semióticos, en términos del profesor Marques (2000):

- Códigos linguísticos: texto, frase, remaargumento, en términos del semiólogo Charles Peirce

- Códigos visuales: icono, indice, cromático y cualisigno

- Componente morfológico de la imagers: movimientos y sonido

- Componente sintáctico de la imagen planos $\mathrm{y}$ angulos

- Componente semántico de la imagen: topos hiperboles, metáforas.

A partir de estas unidarles de análisis, se disenó una matriz para evidenciar el comportamiento de dichas categorias semióticas, instrumento que permitió a la vez su compilación, en dos figuras en las que se visualiza desde el ac, la tendencia de estas niveles en la produocion audiovisual tanto de la región Colombia. 
como de la región internacional. A continuación se aprecia en la Tsus 2 , la matriz ac.

Tabla 2. Matriz análssis de contenido (ac)* en productos audiovisuales, actor Colombia

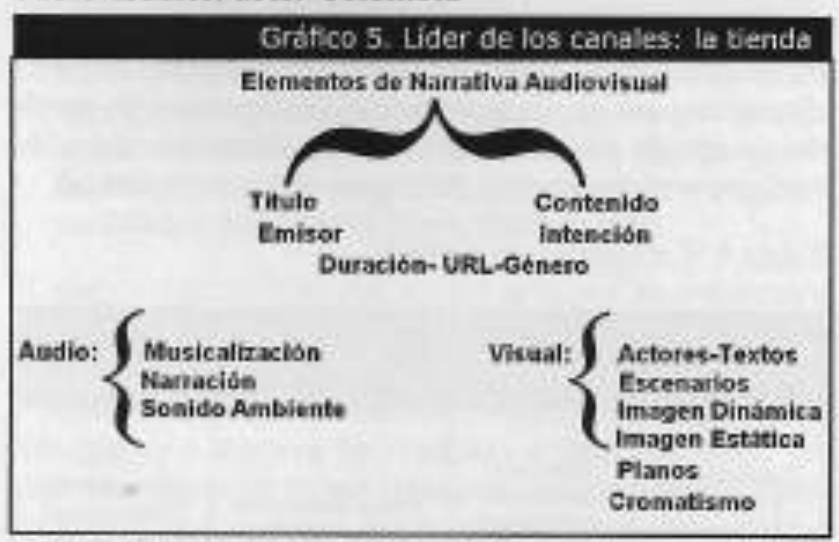

- In términos del Maestro Kripgendorf, entendamos por Analiśs de Canteniclo- AC:- una técnica de inwestigacián que compila información del contenido de un texto escrita pintado, filenado (.). con el propúsito de realizar uma descripcióo objctiva y siscemalira de cierios rasgos especifleos, directamente observables y goe nos persniten flacer inferencias valiosas de conocinicatos $y$ de fencenenos de la vida social (Andreu, 2001).

Fuente: Barón, Mireya, 2010.

A partir de esta matriz, se realizo el $\mathrm{AC}$ en las piezas audiovisuales tanto de la región pais como de la región intemacional, de la cual se compilan los siguientes datos:

- Título del producto audiovisual: - Presentación tailer departamental ruta atención víctimas minas antipersonal", "Las minas antipersonal son inhumanas", "Minas antipersona, soldado perfecto", "El asesino invisible" (crónica ty subida a la red), "FrRC fuerzas armadas revoluciunarias de colombia", 'Minas antipersona, juntos por los Andes". "No más minas, versión reggaeton ladaptación de cancion de Michael Jacksan They don treally care about as", "Una historia de autocuidado para todos". "Presentaciones de educación sobre riesgos ante minas antipersonales por parte de organizaciones de Colombia", "Burma Issues; Thailand Mayanmar Border Mae Sot Refugee Landmine Survivors', 'Los nifios de Arrupe", "Unos zapatos para Casinda", entre otros.
- Emisor región Colombia - región internacional: Cruz Roja Colombiana, Cruz Roja Española, Vicepresidencia de la Republica Colombia, Fundaciones Nacionales. oNG Organizaciones No Gubernamentales : Fundación MI Sangre, Video Defensores y Docentes, Unicef, cpi.org, Landminesanonitor. org, Programadoras de Televisión (ren, Canal 4. Colombia y Niche Films, tv España, pax wori.D Adopt-A-Minefield's Goodwill Ambassadors).

- Componente morfológico (sonido): explosiones, voe narrador adulto- infantil, canciones artistas locales internacionales, música autóctona.

- Código lingúistico ftexto enunciado argumento): "Segün ta Unicef, Colombla ocupa... "Una mina es un artificio explosivo".." "Ten cuidado al caminar..." 'Las minas son..." No podemos caminar por usted, peno si acompanarlo:.." "8.000-10.000 children ane killed or mutllated by landmines every year'

El estudio se complementó con el registro gráfico del comportamiento de codigos y componentes en region Colombia (Figura 1) y en región internacional (Figura 2).

En el sentido de esta caracterización, vale la pena destacar en la muestra "Una historla de autocuidado para todos: elaborada por un docente de Quindio (Colombia), la explicación que hace en el taller a sus estudiantes al explicar las siglas MaP MUSE MNa-minas antipersonal, must-munición sin explotar, se podría decir que este elemento lingüistico se cornvierte en un signo tipo RRsa, que en términos peirceanos, significa que desea enfatizar en el sentido de educación a los estudiantes de básica primaria.

En la Flgura 2 sc Región Internacional, se destaca que las muestras audiovisuales producidas desde Asia, enfatizan en la descripción de la situación problema: sin embargo se alternan muestras audiovisuales en las que la superación por medio de actividades autóctonas (danzas, deportes) evidencia diversos niveles de construcción de sentido acorde con el tipo de contexto del flagelo minas antipersonal. 


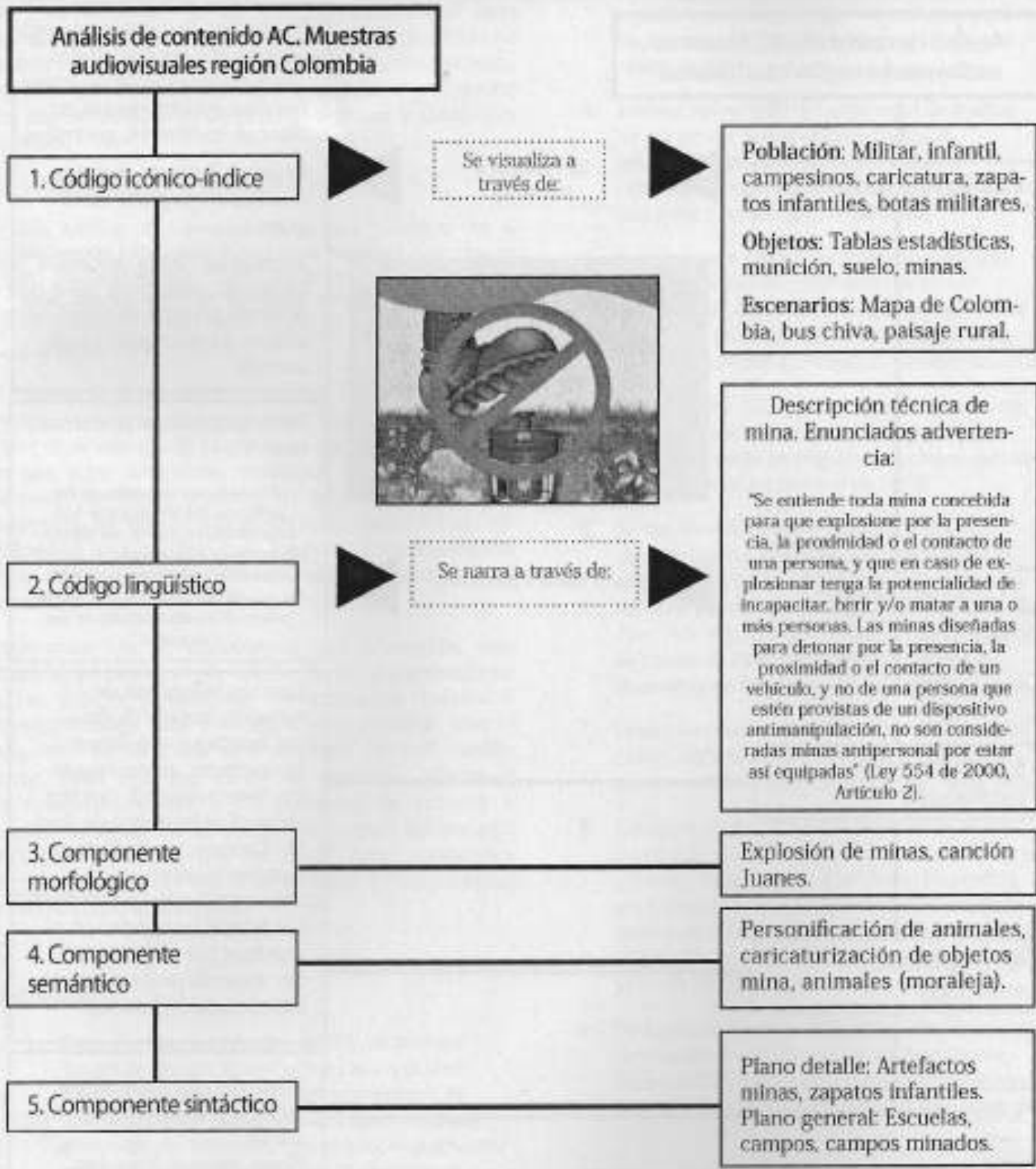




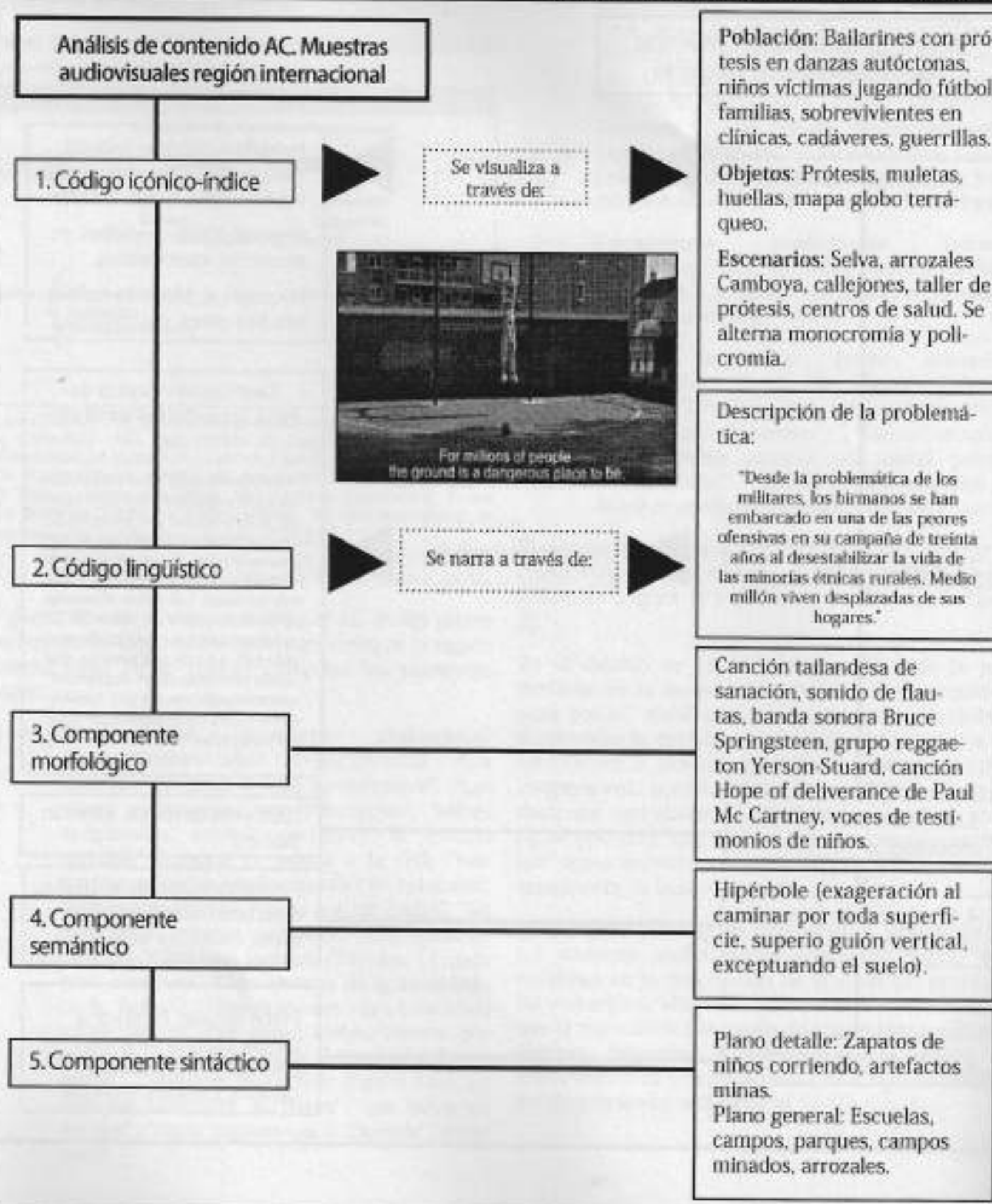




\section{Condusiones}

De este recorrido de la semiosis audiovisual caso minas antipersonal, resulta valiosa la estructuración de dichos relatos destacando el papel de la comunicación narrada con imagen, sonido y movimlento por medio del uso intencionado de planos, ángulos y metáforas visuales.

En esta investigación se destaca que

Los medlos de comutuscactón pueden participar en el desarrollo local colaborindo en ta supcrachin do una de lis Lmataciouses que tsenen lice proyoctos de desarrollo kocal, que es cumo se comunica este proceso para que no quede chocrato en el grupo de genite que lo hace (Corapgin, 2001, p. 12).

Por tanto, la propuesta documental Si no lo boto no lo recoja, que acompaña este texto y que emerge come pieza diseñada desde la woz local regional se convlerte en un actor altamente potencial con el propósito de crear sentido por medlo de la prevención en los contextos de mayor vulnerabilidad, para ol caso de Colombia, sin olvidar que América Latina también tiene una importante población rural virtima de este contexto.

Esperamos que el documental que acompaña este articulo, se divulgue por medio de las arganizaciones y los líderes promotores de entidades locales e internaclonales. Esta estrategla está pensiala con el propósito de fomentar una semiosis para el cambio social, toda vez que es en la selección coherente de referentes afines a los procesos de emisión e interpretación de la audiencia interlocutora del mensaje audiovisual, en los que se construyen contenidos eficaces a partir del cejido de cúdigus y componentes de la narrativa audlovisual"

\section{Bibliografia}

1. Gumucio-Dagron, Alfonso. (2006), mifome del Grupo de Trabuju sabre Comunicacibin para d Cambio Social Asoclación Latinoamericana de Investigación en Comunicación (Alaic) len línea). disponible en:httpd/alaicgtccspooglepages.com/ alaic2006-ponencias (consulta. 18 de mayo de 2010).

4 Para apceciar el docunseztal se pocde ingresar ea hitpl/www. youtube. courv'results? seardi_query-minos+andipersona+siton+lo+b otounn+lorrecujakaq-f
2. Alvarez, E; icaza, Longoria (2000). Mediosy scciedad civit una alianza para el control poübico ten

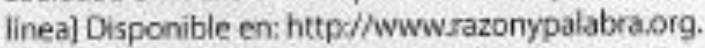
mx/anteriores/nis/18ealvarez,html (consulta: 2 de marzo de 2009).

3. Andreu, Jaime. (2001) Las tecmicas de andllsis de conterido: una revisión actualizada. [en linea] Disponible en; http://public. centrodeestudiosandaluces.es/pd/s/5200103.pdf (consulta: 8 de septiembre de 2010).

4. Barán, Mireya. (2009). Serniosis en el discusso medíficico sobve el cuburmiento de minas antipersonar. Colombla una reaüdad: [en linea] disponible en: http//www:razonypalabra.org. $\mathrm{mw} /$ Mireya_Baron.poff, (consulta: 27 de abril de 2010).

5. Barón, Mireya 12010), si no to botd, no lo recogia. un relato desde el sentir de las regiones. (en Ilnea] Disponible en http://crear.poligraneduco/ (consulta: 8 de septiembre de 2010 ).

6. Barón, Mireya $\{2010\}$. El hombre telacional, comunicación y elementos estratégicos en el discurso de minas antirpesonal, Colombia una realidad. (versiôn electrónica) Revista Electrónica Fisec, Anov (13), p. 13-21. Universidad Nacional de Lomas de Zamora (Argentinal Ien líneal.. disponible en http://www.fisec-estrategias.com.ar/

7. Centre International de Déminage Humanitaire. (2002). Mejorando la comunicacián en lís programas de sensubilzación cantra minas. Genova.

8. Coraggio, José Luks, (2001). El rol sodar de los medios de comumicación y comunicadores en ef contexto focal y regional [en linea] Disponible en: enhttp://www.coraggiceconomia.org/jle/ archivos\%20para\%20descargariDesgrabacinROLSOCIALDELOSMECIOS doC.pof (consulta: 29 de junio de 2010).

9. De Saussure, Ferdinand, (1945) Curso de Linguijstica Genera/ (en linea] Disponible en: http://rwww. liccom.edu.uy/bedelia/cursos/semiotica/textos/ saussure linguistica.pdf (consulta: 4 de mayo de 2010).

10. Dominick-Wimmer, 2001, Wrraducción a la investigacidin de medios masivos de comunicación. Mexicat Thompson. 
11. Farjat, Jorge Luis. 2004, Teond audionsusal E audionisual camo arte extractos de la teona audiovisualogit: el audiovisual como ante y medio de cominicación dentificación y codificacián de la teovia del montaje en la seleccidon iconogrdica Buenos Aires: Gráfica integral.

12. Liceras, A. (2005), Los medias de comumicadion de masas, edincacion informal y aprendizajes sociales. (en linea] Disponible en: www.histodidactica.com, wwwubes/histodidactica (consulta: 6 de mayo de $2010 \%$.

13. McKee, Robert. (2004), El gǘón sustan-ia, estructura, estiolo y amincipios de la escritura de guiones. España Alba.

14. Marques G., Pere. (2000). La alfabetización dudiovisual. [en línea] Disponible en: $h u p d / /$ peremarques.pangea.org/alfasudi.htmAiniciotinicio (consuita: 5 de mayo de 2010).

15. Melone, Sandra, et al. (2002). Vitizzadidn de los medios de comunicactón en la trans famacián de confictos la expeniencla del Comman Ground [en linea] Disponible en: http://www.berghofhandbook.net/documents/publications/spanish_ melone_handbbook.pdf (consulta: 7 de septiembre de 2010):

16. Paole, Antonio, (2002). Comunicaobon y juego simbólica relaciones sociales, cutura y procesos de significación México: Umbral.

17. Peirce, Charles, (2007), La Kógica considerada como semífrica, Madrid: Biblioteca Nueva.

18. Pérez Monter, Héctor Javier (2007). El gươn audiowsual, su estructuws en gentero de ficcidny no ficción y una perspectiva sociocutural. México: Trillas.

19. Ramos, Jesús; Marimón, Joan. (2004). Diccianario dê guiớn. España: Océano Ámbar.

20. Roldản, L; Cárdenas, T. (1994). Teoria y práctica en la producción de un video educativo. En Revista Camunicar (3) p. 43-49 [en linea] Disponible en: http//balboa.unexes/crai/personal/evte/ Comunicar/1994_3/roldan.pdf (consulta: 5 de septiembre de 2010).

21. Severino, Josefina. (2007). Colombis al linite Producción Audiovisual.
22. Vicepresidencia de la República de Colombia. [2007). Observatorio de Minas Antipersonal, Unicef. Guia parte 5 aus fintemational Mine Action Standards- Eorucación en el riesgo de las minas.

\section{Fuentes de documentadón primaria - Páginas web}

1. wwwiderechoshumanos.govico/observatorio_de/ minas.

2. unwuniceforg.co/minas/pub,

3. www.colombiasinminas.org

4. www.accioncontraminas.govico

\section{Muestra online (enlaces vigentes a mayo 6 de 2010)}

5. hitp:/iwww.slidesharenet/accioncontraminas/ presentacin-taller-dptal-ruta-atencin-vctimasminas-antipersonal-presentation

6. http://www youtubecom/

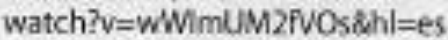

7. http $/$ /www.tu.tv/videos/no-mas-minas-antipersona

8. httpd/wwwyoutube.com/ watch?v=MDiPp2ZjvGo\&hl=es

9. http//www.youtube.com/watch?v=0NSodyld lik\&N $R=18$ feature $=$ fwwp

10. http//wwwlukcr.com/videas/minas: antipersonales- $/ \times 7$ ohQ-TWGi\&feature =youtube

11. http://www youtubecom/watch?v=VzmReA0L.pKg

12. http//wwwlukor.com/videos/minas-antipersona-/ fbas_6p50jsafeature=youtube_gdata/

13. http//Www.youtube.com/watch/v=loKStTOg06 M

14. http//wwwyoutube.com/results?search quer $y=m i n a s+a n t i p e r s o n a s+e n+c o m o l i m b i a \& s e a r c$ ha_type $=8 \mathrm{ac}=\mathrm{f}$

15. http $/ /$ wwwicrcorg/WEB/SPA/sitespa0.ns//htmlall/ owym-video-colombia-landmines?OpenOocumen

16. $h t t p: / / w w w . y o u t u b e . c o m / w a t c h ? v=V z m R e A 0 L p K g$ 